\title{
BAYESIAN ANALYSIS OF RESERVING MODELS AND APPLICATIONS
}

\author{
X. D. A. DONG \\ (Received 15 November 2015; first published online 25 July 2016)
}

2010 Mathematics subject classification: primary 62C12; secondary 62F15, 97M30.

Keywords and phrases: Bayesian, loss reserving, GB2, quantile regression, copula.

A Bayesian approach is presented in order to model long tail loss reserving data using the generalised beta distribution of the second kind (GB2) with dynamic mean functions and mixture model representation. The proposed GB2 distribution provides a flexible probability density function, which nests various distributions with light and heavy tails, to facilitate accurate loss reserving in insurance applications. Extending the mean functions to include the state space and threshold models provides a dynamic approach to allow for irregular claims behaviours and legislative change which may occur during the claims settlement period. The mixture of GB2 distributions is proposed as a means of modelling the unobserved heterogeneity which arises from the incidence of very large claims in the loss reserving data. It is shown through both simulation study and forecasting that model parameters are estimated with high accuracy.

Apart from estimating the mean, we also develop quantile regression models in order to derive risk margin and to evaluate capital in non-life-insurance applications. By utilising the entire range of conditional quantile functions, especially higher quantile levels, we detail how quantile regression is capable of providing an accurate estimation of risk margin and an overview of implied capital based on the historical volatility of a general insurer's loss portfolio. Two modelling frameworks are considered based around parametric and nonparametric quantile regression models, which we develop specifically in this insurance setting.

In the parametric quantile regression framework, several models including the flexible generalised beta distribution family, asymmetric Laplace (AL) distribution and power Pareto distribution are considered under a Bayesian regression framework. The Bayesian posterior quantile regression models in each case are studied via Markov

Thesis submitted to the University of Sydney in August 2015; degree approved on 25 September 2015; supervisors Jennifer Chan and Gareth Peters.

(c) 2016 Australian Mathematical Publishing Association Inc. 0004-9727/2016 \$16.00 
chain Monte Carlo (MCMC) sampling strategies. In the nonparametric quantile regression framework, which we contrast to the parametric Bayesian models, we adopted an AL distribution as a proxy and together with the parametric AL model we expressed the solution as a scale mixture of uniform distributions to facilitate implementation. The models are extended to adopt dynamic mean, variance and skewness and applied to analyse two real loss reserve data sets to perform inference and discuss interesting features of quantile regression for risk margin calculations.

Finally, we consider the class of recently developed stochastic models that combine claims payments and incurred losses information into a coherent reserving methodology. In particular, we develop a family of hierarchical Bayesian paidincurred-claims models. In the process we extend the independent log-normal model by incorporating different dependence structures using a data-augmented mixture copula paid-incurred-claims model.

The utility and influence of incorporating both payment and incurred losses into estimating the full predictive distribution of the outstanding loss liabilities and the resulting reserves is demonstrated in the following cases: (i) an independent payment (P) data model; (ii) the independent payment-incurred-claims (PIC) data model; (iii) a novel dependent lag-year telescoping block diagonal Gaussian copula PIC data model incorporating conjugacy via transformation and (iv) a novel data-augmented mixture Archimedean copula dependent PIC data model. Inference in such models is developed via a class of adaptive MCMC sampling algorithms. These incorporate a data-augmentation framework utilised to efficiently evaluate the likelihood for the copula-based PIC model in the loss reserving triangles.

\section{D. A. DONG, School of Mathematics and Statistics, The University of Sydney, NSW 2006, Australia e-mail: alice.dong10@gmail.com}

